\title{
Novel active method for the estimation of a building wall thermal resistance
}

\author{
Adrien François ${ }^{1,2^{*}}$, Laurent Ibos $^{1}$, Vincent Feuillet ${ }^{1}$ and Johann Meulemans ${ }^{2}$ \\ ${ }^{1}$ Univ Paris Est Creteil, CERTES, F-94010 Creteil, France \\ ${ }^{2}$ Saint-Gobain Research Paris, 39 quai Lucien Lefranc, 93300 Aubervilliers, France
}

\begin{abstract}
The thermal resistance of a wall can be readily measured in steady-state. However, such a state is seldomly achieved in a building because of the variation of outdoor conditions as well as the high thermal inertia of building materials. This paper introduces a novel active (dynamic) method to measure the thermal resistance of a building wall. Not only are active approaches less sensitive to external temperature variations, they also enable to perform measurements within only a few hours. In the proposed methodology, an artificial thermal load is applied to a wall (heating of the indoor air) and its thermal response is monitored. Inverse techniques are used with a reduced model to estimate the value of the thermal resistance of a wall from the measured temperatures and heat fluxes. The methodology was validated on a known load-bearing wall built inside a climate chamber. The results were in good agreement with reference values derived from a steadystate characterization of the wall. The method also demonstrated a good reproducibility.
\end{abstract}

\section{Introduction}

From the perspective of limiting greenhouse effect, two approaches have to be considered: the development of the use of renewable energies and the limitation of global energy consumption. Regarding the second aspect, the building sector represents a significant part of the world primary energy consumption. Following the national building regulations implemented by governments, the thermal resistance of buildings walls tends to increase. However, a non-negligible difference is usually observed between theoretical estimations and in situ measurements of the thermal performance of buildings: this often referred to as the "performance gap" [1]. One major cause of the performance gap in practice is thermal bridging.

Several methods were developed in academia and a lot of efforts are currently being made to improve their accuracy, rapidity and applicability. Bienvenido-Huertas et al. recently published a comprehensive review [2] summarizing most of these methods. For the sake of completeness, let us add the QUB/e method [3] to this review. However, these methods are deemed not mature enough to be widely used by the building construction industry. For the thermal insulation of building walls, there are only two existing standardized techniques, namely ISO 9869-1 [4] and 9869-2 [5]. Nevertheless, they do not accurately evaluate a thermal resistance in many situations, especially if heat transfers are far from being in steady-state regime and if the indoor-outdoor temperature gradient is too small. This is mainly because these approaches are passive methods, therefore strongly influenced by climate conditions.
Active methods are an interesting alternative. They consist in applying an artificial thermal load to a wall and analysing its thermal response. The excitation may be of different nature: radiant heater, sun power, air heating, etc. Active methods have the advantage of being less sensitive to non-constant climate conditions. They are also faster than passive methods.

The present study introduces a novel active in situ measurement of a wall thermal resistance. The indoor air is heated with electric heaters and the wall surface temperature as well as the heat flux are measured with contact sensors. Inverse methods are then applied to these measurements in order to estimate the wall thermal resistance from the dynamic thermal response of the wall. The direct model used in the inverse techniques must have a complexity adapted to the problem. The methodology was validated on a wall built inside a climate chamber. This wall is made of concrete building blocks on which a conventional internal insulation system is fixed.

This paper is composed of 3 sections. Section 2 presents the experimental setup used to validate the method. Section 3 details the inverse method used to estimate the wall thermal resistance. Finally, results are presented and discussed in Section 4.

\section{Experimental setup}

\subsection{Presentation}

The measurements were performed on a load-bearing wall built inside a $4 \times 4 \times 3 \mathrm{~m}^{3}$ climate chamber at CEREMA (Centre d'Etude et d'Expertise sur les Risques, l'Environnement, la Mobilité et l'Aménagement) in Nancy, France. As shown in Figure 1, the chamber is

\footnotetext{
* Corresponding author: adrien.francois@u-pec.fr
} 
equipped with two independent modules able to control the temperature between -30 and $30^{\circ} \mathrm{C}$. The wall was built between the two modules so that a different temperature could be set on each side.

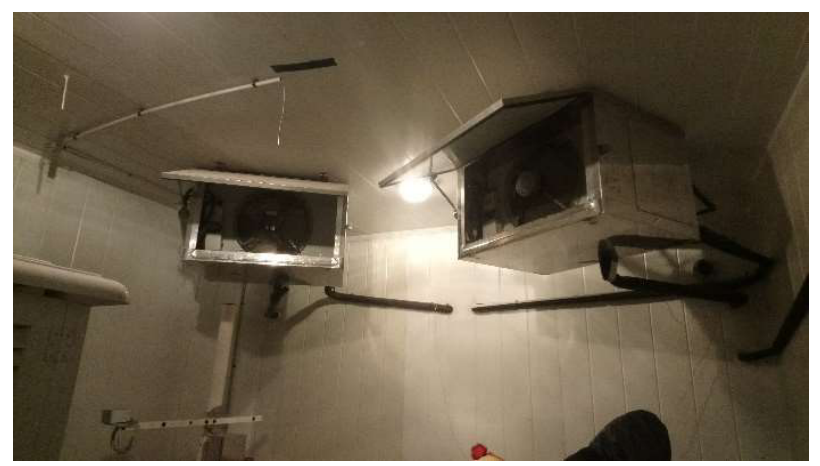

Fig. 1. Modules inside the climate chamber

The wall is $3.2 \mathrm{~m}$ wide and $2 \mathrm{~m}$ high and a thin door on the right-hand-side enables to go behind it. Figure 2 shows a photography of the wall before the installation of the right-hand-side gypsum board.

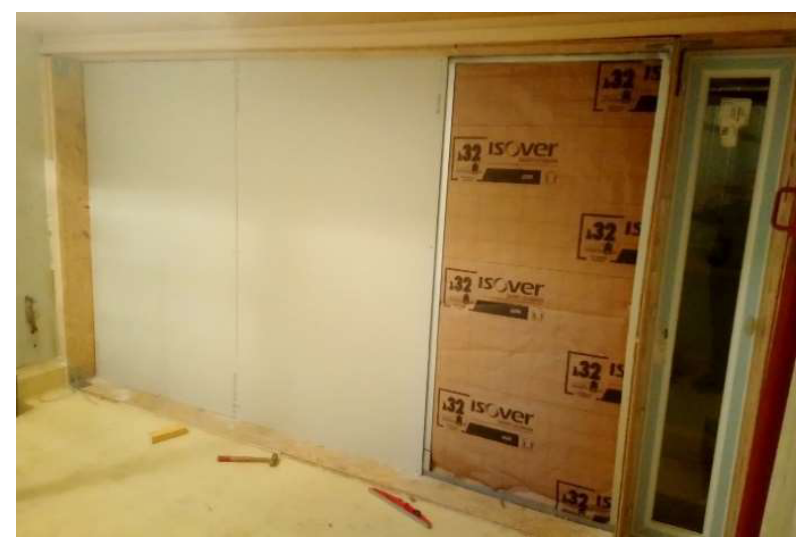

Fig. 2. Photography of the wall (without one gypsum board)

It consists of three layers (see Figure 3). The first one is made out of $20 \mathrm{~cm}$-thick concrete building blocks. Then, a conventional internal insulation system was fixed: 10 $\mathrm{cm}$ of glass wool held with metal rails and covered by a gypsum board.

The gypsum board in the middle of the wall is only held by metal rails on its edges. The absence of rails in its centre enables to benefit from a large zone (1.2 m wide) where heat transfers are 1D (i.e., without any thermal bridge). Indeed, the metal rails makes the transfers $2 \mathrm{D}$ or 3D which complexifies the problem. An infrared camera was used to check the temperature uniformity on the centre of the wall.

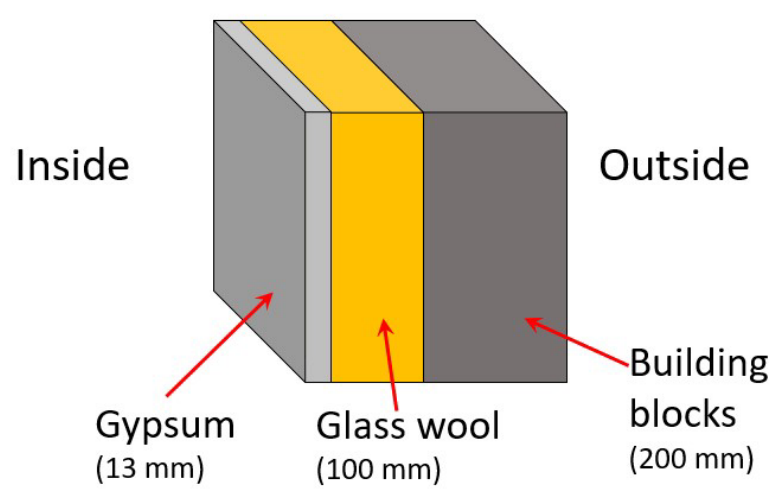

Fig. 3. Scheme of the wall cut-out

\subsection{Theoretical values}

From knowledge of the material thermal conductivity $\lambda$ of the materials and the thickness $d$ of the layers, the theoretical thermal resistance of the wall can be calculated:

$$
R=\frac{d_{1}}{\lambda_{1}}+\frac{d_{2}}{\lambda_{2}}+\frac{d_{3}}{\lambda_{3}}
$$

It may be noted that contact resistances between materials are neglected. The thermal conductivity of the gypsum was measured in laboratory with the "Hot Disk" method [6]. For the glass wool, manufacturer's data was used. The thermal properties of the insulation layers are reported in Table 1.

Table 1. Thermal properties of the insulation layers

\begin{tabular}{|c|c|c|}
\hline Layer & gypsum & glass wool \\
\hline$d(\mathrm{~m})$ & 0.013 & 0.1 \\
\hline$\left(\mathrm{W} \cdot \mathrm{m}^{-1} \cdot \mathrm{K}^{-1}\right)$ & 0.23 & 0.032 \\
\hline $\boldsymbol{R}\left(\mathbf{W} \cdot \mathbf{m}^{-\mathbf{1}} \cdot \mathbf{K}^{-\mathbf{1}}\right)$ & $\mathbf{0 . 0 6}$ & $\mathbf{3 . 1 2}$ \\
\hline
\end{tabular}

Thus, the thermal resistance of the insulation system (gypsum + glass wool) is:

$R_{\text {theo }}=3.18 \mathrm{~m}^{2} \cdot \mathrm{K} \cdot \mathrm{W}^{-1}$

The associated uncertainty is estimated to $0.15 \mathrm{~m}^{2} . \mathrm{K} . \mathrm{W}^{-1}$.

\subsection{Sensors and data acquisition}

Temperatures were measured with $0.2 \mathrm{~mm}$-thick type-T thermocouples. These sensors were calibrated between 0 and $60^{\circ} \mathrm{C}$ with a platinum sensor which enabled to reach an uncertainty of $0.1^{\circ} \mathrm{C}$. In addition, heat flux densities were measured using normal gradient heat flux meters (HFM). These $10 \times 10 \mathrm{~cm}^{2}$ sensors are from Captec ${ }^{\odot}$ manufacturer. A type-T thermocouple is also embedded inside. They have a sensitivity greater than $60 \mu \mathrm{V} /\left(\mathrm{W} / \mathrm{m}^{2}\right)$ 
which is given with a $3 \%$ accuracy. The surface of the HFMs were covered with an adhesive tape which has similar optical properties as the wall surface: same emissivity (0.94 in the $2-20 \mu \mathrm{m}$ band) and diffuse reflection. This allows the sensors to be as less intrusive as possible. Temperature and heat fluxes were continuously monitored every $3 \mathrm{~s}$ and averaged every $15 \mathrm{~s}$.

\section{Methodology}

Here are presented the methodologies used to measure the wall thermal resistance both in passive and in active configurations.

\subsection{Passive characterization}

Steady-state measurements were undertaken to obtain a reference thermal resistance of the wall. This value will serve as a reference that will be useful to assess the accuracy of the active method presented in the next section. The measurements were conducted according to standard ISO 9869-1 [4]. The external and internal temperatures were set to constant values: 5 and $25^{\circ} \mathrm{C}$ respectively so that a temperature difference of $20^{\circ} \mathrm{C}$ was ensured across the wall (on the area free of thermal bridge). The wall required about 3 days to reach thermal equilibrium. A heat flux meter was placed on the middle of the wall to record the heat flux densities while thermocouples were put on each side as well as between the insulation system and the building blocks. The measured quantities were averaged over a period of two hours. This procedure filters out the high frequency temperature variations due to the regulating system of the climate chamber. The measured thermal resistance is given by:

$$
R=\frac{\Delta T}{q}
$$

With $\Delta T$ a surface temperature difference and $q$ the heat flux across the wall.

The measurement uncertainty was propagated from the uncertainty associated to $\Delta T$ and $q$ [7]

$$
u_{R}=\sqrt{\left(\frac{\partial R}{\partial \Delta T} u_{\Delta T}\right)^{2}+\left(\frac{\partial R}{\partial q} u_{q}\right)^{2}}
$$

This leads to the following result:

$$
R_{\text {passive }}=3.15 \pm 0.06 \mathrm{~m}^{2} \cdot \mathrm{K} \mathrm{W}^{-1}
$$

This does not include the thermal resistance of the concrete blocks: $\Delta T$ was measured between the indoor surface and the surface between the glass wool and the blocks. This result is in good agreement with theoretical predictions: the relative difference is of $1 \%$. Its uncertainty is also smaller.

\subsection{Active characterization}

\subsubsection{Thermal load}

Active methods rely on an artificial thermal load. Here, it was chosen to heat up the indoor air. This approach has the advantage of being easily applicable in situ thanks to electric heaters. In addition, it enables to heat up the entire wall rather than only a small area. This artificial thermal load is also used in the QUB/e method [3].

In practice, the temperature regulation of the climate chamber is switched off on the indoor side and two $500 \mathrm{~W}$ electric heaters are switched on for a duration of about $8 \mathrm{~h}$. Meanwhile, the temperature on the other side is controlled and remains constant. The indoor and outdoor initial air temperatures are $5^{\circ} \mathrm{C}$ and $15^{\circ} \mathrm{C}$ respectively. The air temperatures measured with thermocouples on each side of the wall during an active test are plotted in Figure 4.

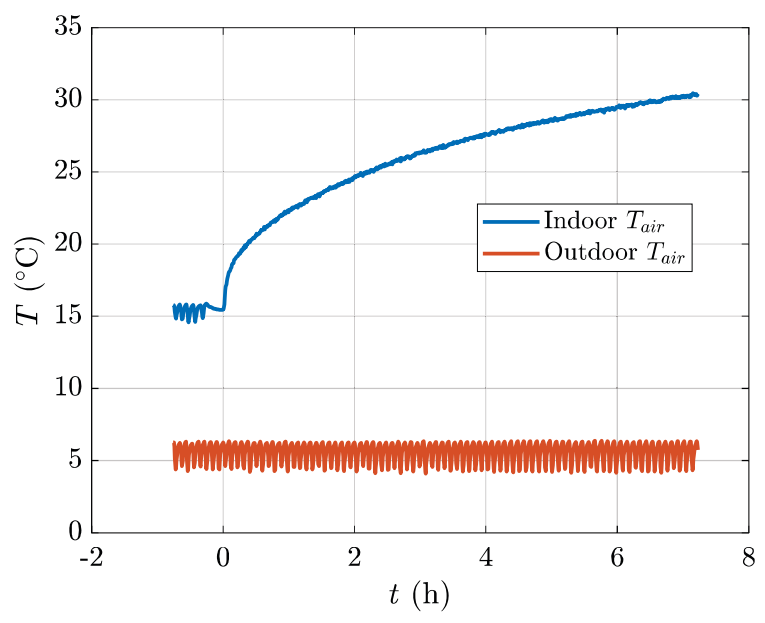

Fig. 4. Air temperatures during an "active" experiment

Time $t=0$ corresponds to the start of the electric heaters. The apparent noise on the outdoor temperature as well as at the beginning on the indoor temperature are due to air temperature fluctuations induced by the regulation systems of the climate chamber.

It is important to point out that these two temperatures will not be used for the estimations of the wall thermal resistance (see sections 3.2.5 and 3.2.6). Thus, the exact location where there were measured is not important here.

\subsubsection{Measured quantities}

A heat flux meter plate is mounted on the sound area (no thermal bridge) of the indoor surface, $1.4 \mathrm{~m}$ above the ground. It measures both the surface heat flux densities $\varphi_{s i}$ and temperature $T_{s i}$ thanks to the embedded thermocouple. The time evolution of these quantities is plotted in Figure 5. 


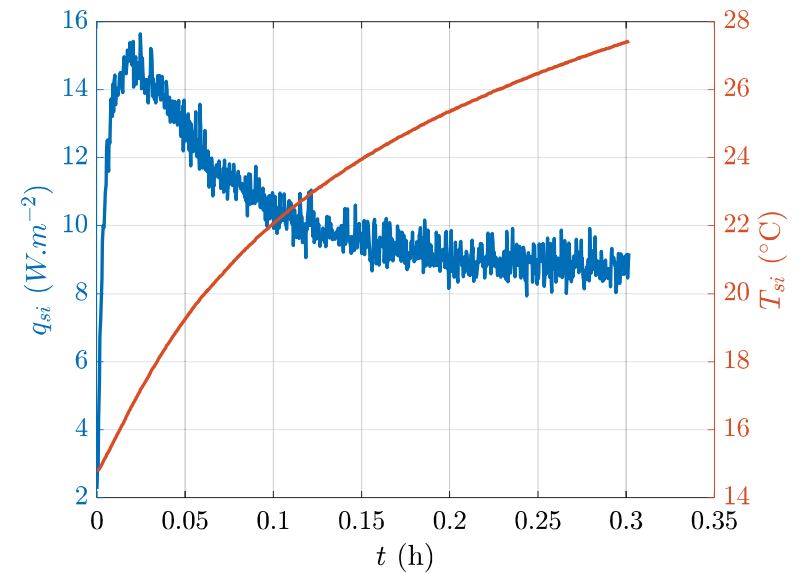

Fig. 5. Measured surface temperature and heat flux

It may be noticed that the signal to noise ratio (SNR) is much better for the temperature than for the heat flux measurement.

\subsubsection{Presentation of inverse methods}

The wall thermal resistance is estimated from $T_{s i}$ and $q_{s i}$ using an inverse technique. It is a non-linear parameter estimation problem [8]. Basically, we first consider the "direct problem". It is defined by a model $y_{m o}$ that takes some parameters as input (gathered in a vector $\boldsymbol{\beta}$ ) and gives an observation $y$ as output. In the present context, $\boldsymbol{\beta}$ is composed of the wall thermal characteristics (thermal conductivities, thicknesses, etc.) and the observation $y$ is the heat flux $q_{s i}$ on the indoor surface of the wall (the motivations of this choice are detailed below). However, we are not interested here in calculating the surface heat flux from the wall characteristics. We rather want to do the exact opposite: estimate the wall characteristics $\boldsymbol{\beta}$ from measurement of the surface heat flux, hence the terminology "inverse method".

The parameters are estimated by minimizing the cost function $J$ that quantifies the difference between the model and the measurements:

$$
J=\sum_{i=1}^{m}\left(q\left(t_{i}\right)-q_{m o}\left(\boldsymbol{\beta}, t_{i}\right)\right)^{2}
$$

with $m$ the number of points.

It is important to point out that the estimation of the desired parameters is not straightforward. Indeed, inverse problems are often ill-posed, and the choice of the experiment as well as the definition of the direct model are crucial. A too simple direct model would not be able to capture the physical phenomenon investigated while a too complex model would have too many parameters which would make the estimation impossible or biased.

\subsubsection{Thermal modelling and parameterization}

The problem considered is supposed one-dimensional. It is illustrated in Figure 6. Each layer is characterized by its thermal resistance $R$ and thermal effusivity $b$. With the two heat exchange coefficients $h_{i}$ and $h_{e}$, the inverse problem has eight unknown parameters.

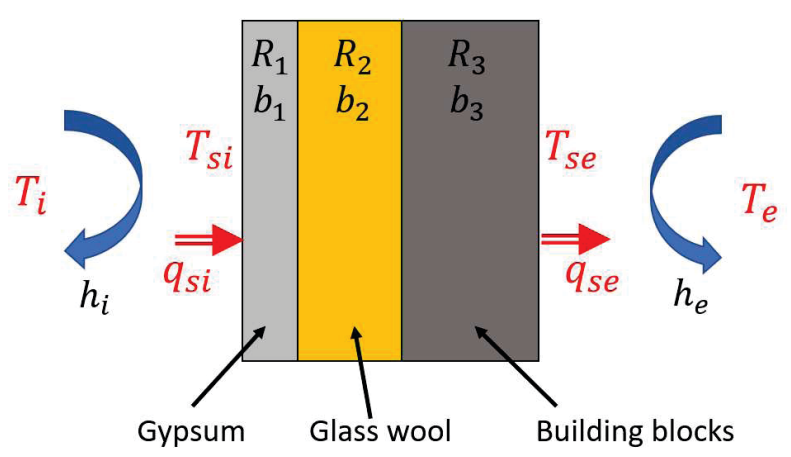

Fig. 6. Illustration of the complete problem (8 unknown parameters)

The direct model is derived from the thermal quadrupole formalism [9]. It is derived from the Laplace transform of the one-dimensional heat equation. The advantage is that the relationship between the Laplace transforms of temperatures $\bar{T}$ and heat fluxes $\bar{q}$ is simply given by a matrix multiplication:

$$
\left[\begin{array}{l}
\overline{T_{s i}} \\
\overline{q_{s i}}
\end{array}\right]=M\left[\begin{array}{l}
\overline{T_{s e}} \\
\overline{q_{s e}}
\end{array}\right]
$$

with $M$ a $2 \times 2$ matrix.

Each homogeneous layer $k$ of the wall is characterized by the following quadrupole matrix:

$$
M_{k}=\left[\begin{array}{cc}
\cosh \left(R_{k} b_{k} \sqrt{p}\right) & \frac{\sinh \left(R_{k} b_{k} \sqrt{p}\right)}{b_{k} \sqrt{p}} \\
\sinh \left(R_{k} b_{k} \sqrt{p}\right) \times b_{k} \sqrt{p} & \cosh \left(R_{k} b_{k} \sqrt{p}\right)
\end{array}\right]
$$

with $p$ the Laplace variable, $R_{k}$ and $b_{k}$ the $\mathrm{k}^{\text {th }}$ layer thermal resistance and effusivity. In the case of a multilayer wall, the quadrupole matrix $M$ is simply given by the multiplication of matrices $M_{k}$.

The internal and external heat exchange coefficients $h_{i}$ and $h_{e}$ are also included in the model thanks to a $2 \times 2$ matrix. For $h_{i}$ (and similarly for $h_{e}$ ):

$$
H_{i}=\left[\begin{array}{cc}
1 & 1 / h_{i} \\
0 & 1
\end{array}\right]
$$

Finally, it comes:

$$
\left[\begin{array}{l}
\overline{T_{i}} \\
\overline{q_{i}}
\end{array}\right]=H_{i} M_{1} M_{2} M_{3} H_{e}\left[\begin{array}{l}
\overline{T_{e}} \\
\overline{q_{e}}
\end{array}\right]
$$

Once the thermal problem is given in the Laplace domain, the De Hoog [10] algorithm is used to numerically work out the inverse Laplace transform and generate data in the time domain.

\subsubsection{Thermal load in the model}

To use the thermal quadrupole formalism, one needs to have an analytical expression of the thermal load in the Laplace domain. In the reduced model, the thermal load is the surface temperature. Therefore, a function of known Laplace transform is fitted on $T_{s i}$. This function does not need to have any physical meaning, it only must fit the 
measured data as well as possible on the whole measurement duration. The following function was chosen:

$$
T(\boldsymbol{\alpha}, t)=\sum_{k=1}^{n} \alpha_{k} t^{1 / k}
$$

Its Laplace transform is given by:

$$
\bar{T}(\boldsymbol{\alpha}, p)=\sum_{k=1}^{n} \alpha_{k} \frac{\Gamma\left(\frac{1}{k}+1\right)}{p^{\frac{1}{k}+1}}
$$

with $\boldsymbol{\alpha}$ a set of parameters to find and $\Gamma$ the gamma function. The order $n$ was empirically set to 7 . It was noticed that a lower order would increase the difference between the function and the measurements while a higher order would not be beneficial. The $\boldsymbol{\alpha}$ coefficients were estimated with the Levenberg-Marquardt algorithm.

\subsubsection{First model reduction}

The direct model given in Figure 6 has eight parameters: $h_{i}, R_{1}, b_{1}, R_{2}, b_{2}, R_{3}, b_{3}$ and $h_{e}$. This is too many for a correct estimation of the wall thermal resistance. As detailed below, the number of degrees of freedom of the problem is lower than eight, so the number of parameters has to be reduced accordingly.

Given the considered wall and the chosen thermal load, some parameters should not be included in the model: several model reduction steps are required.

Not only is the third layer far away from the measurement point (on the front surface), it also has a massive thermal inertia (concrete building blocks). This means that during the few hours of the active experiment, the thermal wave does not have enough time to go through this last layer. Therefore, $h_{e}$ is not modelled and the third layer is treated as a semi-infinite medium. Such a medium is characterized in the Laplace domain by an impedance $Z_{\text {inf }}=1 /\left(b_{\text {inf }} \sqrt{p}\right)$ which depends on the material thermal effusivity now referred as $b_{\text {inf }}$. Parameters $h_{e}$ and $R_{3}$ are removed from the model. Thus, it will not be possible to estimate the thermal resistance of the concrete wall. This is not an issue as the main interest lays in the characterization of the internal insulation system.

The heat exchange coefficient $h_{i}$ is complex to measure and so it the internal temperature $T_{i}$. Indeed, $T_{i}$ is the so-called "operative temperature". It is a weighted average between the air temperature and the mean radiant temperature. In the building sector, the common assumption is to suppose $T_{i}$ equal to the air temperature. This hypothesis is justified in well insulated buildings when passive methods are used. In the current configuration however, the air is rapidly heated, and its temperature can be far from the mean radiant temperature. Thus, is has been chosen to measure both the temperature and the heat flux on the wall surface. This enables to exclude $T_{i}$ and $h_{i}$ from the model.

Finally, the system in Equation 8 reduces to:

$$
\overline{q_{s i}}=\frac{C Z_{i n f}+D}{A Z_{i n f}+B} \overline{T_{s i}}
$$

with

$$
\left[\begin{array}{ll}
A & B \\
C & D
\end{array}\right]=M=M_{1} M_{2}
$$

This model has 5 parameters: $R_{1}, b_{1}, R_{2}, b_{2}$, and $b_{\text {inf }}$. If $T_{s i}$ is known, $q_{s i}$ may be calculated. In the present study, $T_{s i}$ is supposed to be known (plays the role of the thermal load) and $q_{s i}$ is the output of the model.

\subsubsection{Second model reduction: sensitivity analysis}

The model has been reduced to 5 parameters. A sensitivity analysis is necessary to assess the ill-posedness of the problem and to determine whether the parameters can be estimated with an inverse method.

The reduced sensitivity coefficients quantify how much the model output depends on each parameter $\beta_{j}$. They are simply given by the partial derivative of the output according to $\beta_{j}$ :

$$
\boldsymbol{X}\left(\widehat{\beta}_{j}\right)=\frac{\partial \boldsymbol{q}_{\mathrm{mo}}}{\partial \beta_{j}}
$$

with the ${ }^{\wedge}$ subscript referring to estimated values.

It is more convenient to work with the reduced sensitivity coefficients $X^{*}$ because they are all expressed in the unit of the output which makes them comparable quantitatively:

$$
\boldsymbol{X}^{*}\left(\widehat{\beta}_{j}\right)=\widehat{\beta}_{j} \frac{\partial \boldsymbol{q}_{\mathrm{mo}}}{\partial \beta_{j}}
$$

The evolution of the reduced sensitivities $X^{*}$ with time is plotted in Figure 7.

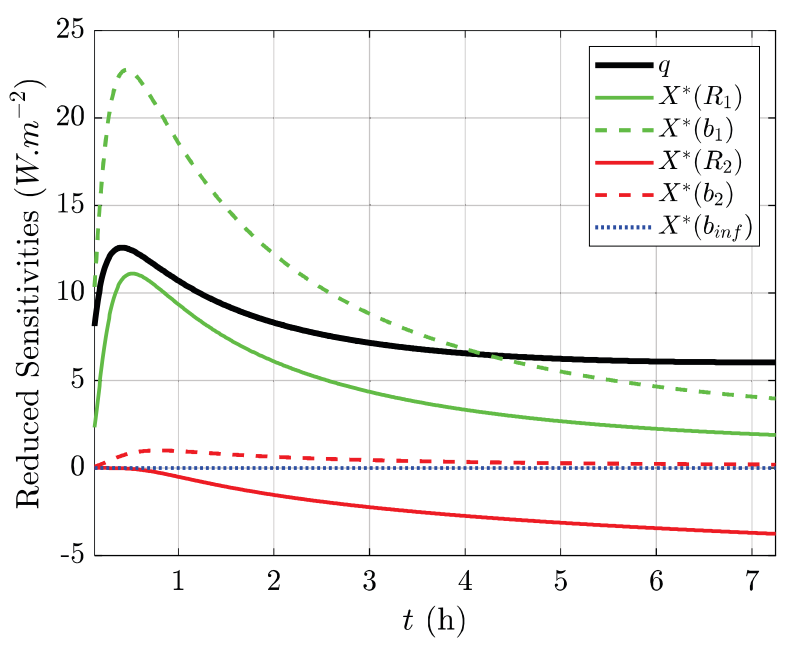

Fig. 7. Reduced sensitivity coefficients of the reduced model

In non-linear problems as the one encountered here, sensitivities are defined locally around nominal values of the parameters. The nominal values used for the present analysis are gathered in Table 2. 
Table 2. Nominal values of parameters

\begin{tabular}{|c|c|c|}
\hline Parameter & Unit & Nominal value \\
\hline$R_{1}$ & $\mathrm{~m}^{2} \cdot \mathrm{K} \cdot \mathrm{W}^{-1}$ & 0.06 \\
\hline$b_{1}$ & $\mathrm{~J} \cdot \mathrm{K}^{-1} \cdot \mathrm{m}^{-2} \cdot \mathrm{s}^{-1 / 2}$ & 420 \\
\hline$R_{2}$ & $\mathrm{~m}^{2} \cdot \mathrm{K} \cdot \mathrm{W}^{-1}$ & 3.12 \\
\hline$b_{2}$ & $\mathrm{~J} \cdot \mathrm{K}^{-1} \cdot \mathrm{m}^{-2} \cdot \mathrm{s}^{-1 / 2}$ & 25 \\
\hline$b_{\text {inf }}$ & $\mathrm{J} \cdot \mathrm{K}^{-1} \cdot \mathrm{m}^{-2} \cdot \mathrm{s}^{-1 / 2}$ & 1500 \\
\hline
\end{tabular}

The first thing to point out is that the sensitivity to the thermal effusivity of the last layer $b_{\text {inf }}$ is negligible during the duration of the experiment. This means that this parameter has no influence on the output $q_{m o}$ and cannot be accurately estimated by inversion. It will therefore be supposed known and fixed to a constant value. Given its very low sensitivity, an error on the default value would have a very limited impact on the estimation.

In the context of the control of a new building for instance, the values given to the supposed known parameters could be supplied by the manufacturer or one could use standard values for the considered materials.

In the end, the reduced model only has four remaining parameters: $R_{1}, b_{1}, R_{2}$, and $b_{2}$, as illustrated in Figure 8:

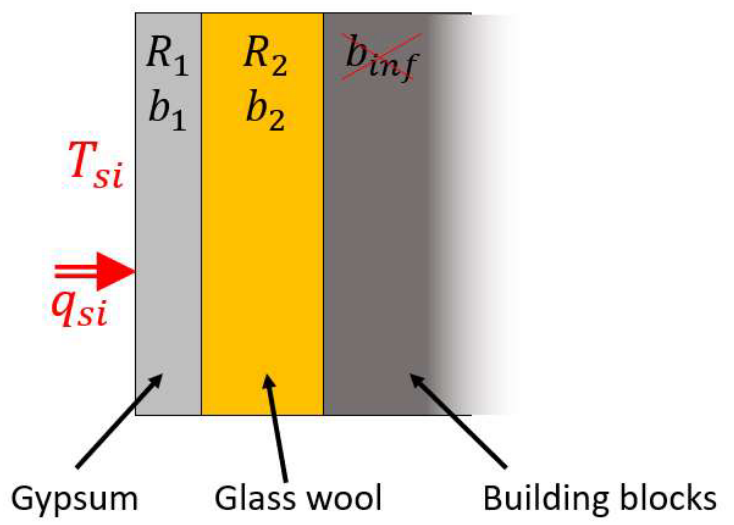

Fig 8. Illustration of the reduced model (4 parameters)

The quantity of interest is $R_{1}+R_{2}$ (thermal resistance of the internal insulation system), and especially $R_{2}$ because $R_{1}$ is negligible. The others $\left(b_{1}\right.$ and $\left.b_{2}\right)$ will also be estimated but their final value is of little interest here. The shape of the reduced sensitivity curve of $R_{2}$ is very different from the one of the other parameters so correlations should be small (this is confirmed below with the Vcor matrix). It may also be noted that the sensitivity to $R_{2}$ increases with time, so there is a minimum duration of the experiment for the estimation of $R_{2}$ to be accurate.

\subsubsection{Covariance matrix and estimation uncertainty}

The measurements are assumed to be corrupted with a white noise of zero mean and of constant standard deviation $\sigma$. The variance-covariance matrix of the inverse problem is derived from the reduced sensitivity matrix $X^{*}$ (see Equation 13) [11]:

$$
\operatorname{cov}=\sigma^{2}\left(X^{* T} X^{*}\right)^{-1}
$$

and

$$
\mathbf{c o v} \approx\left[\begin{array}{ccc}
\operatorname{var}\left(\hat{\beta}_{i}\right) & \operatorname{cov}\left(\hat{\beta}_{i}, \hat{\beta}_{j}\right) & \ldots \\
\operatorname{cov}\left(\hat{\beta}_{i}, \hat{\beta}_{j}\right) & \operatorname{var}\left(\hat{\beta}_{j}\right) & \cdots \\
\vdots & \vdots & \ddots
\end{array}\right]
$$

Let introduce the Vcor matrix:

$$
\operatorname{Vcor} \approx\left[\begin{array}{ccc}
\sqrt{\operatorname{var}\left(\hat{\beta}_{i}\right)} / \hat{\beta}_{i} & \rho_{i j} & \cdots \\
\rho_{i j} & \sqrt{\operatorname{var}\left(\hat{\beta}_{j}\right)} / \hat{\beta}_{j} & \cdots \\
\vdots & \vdots & \ddots
\end{array}\right]
$$

with $\rho_{i j}$ the correlation coefficient between two parameters $\beta_{i}$ and $\beta_{j}$.

These coefficients are between -1 and +1 : the closer to -1 or +1 , the more correlated the parameters. They are an interesting tool to detect ill-posed problems due to correlations between parameters. However, there might also exist correlations between three or more parameters. The diagonal terms of Vcor are the relative uncertainties on estimated parameters.

Using the reference values given in Table 2 and the noise level observed on measurements, the Vcor is calculated.

$$
\begin{array}{c|cccc} 
& R_{1} & b_{1} & R_{2} & b_{2} \\
\hline R_{1} & 0.13 & -0.98 & -0.36 & -0.70 \\
\text { Vcor }= & & 0.06 & 0.42 & 0.60 \\
b_{1} & & & 0.02 & 0.54 \\
R_{2} & & & & 0.10 \\
b_{2} & & & &
\end{array}
$$

Thanks to the model reduction described in the previous section, the parameters are not very much correlated: only $R_{1}$ and $b_{1}$ have a strong correlation coefficient but their estimation is not important. The thermal resistance $R_{2}$ is estimated with an uncertainty of $2 \%$. The relative uncertainties are higher for the other parameters. This is explained by correlations $\left(R_{1}\right.$ and $\left.b_{1}\right)$ and small magnitude of uncertainties $\left(b_{2}\right)$

It is also important to point out that these uncertainties only consider the variance due to the measurement noise (statistical errors). They do not consider any systematic error on the measurement of heat flux $q_{s i}$ or temperature $T_{s i}$. 


\subsubsection{Summary of the methodology}

The methodology described in this section is summarized in Figure 9.

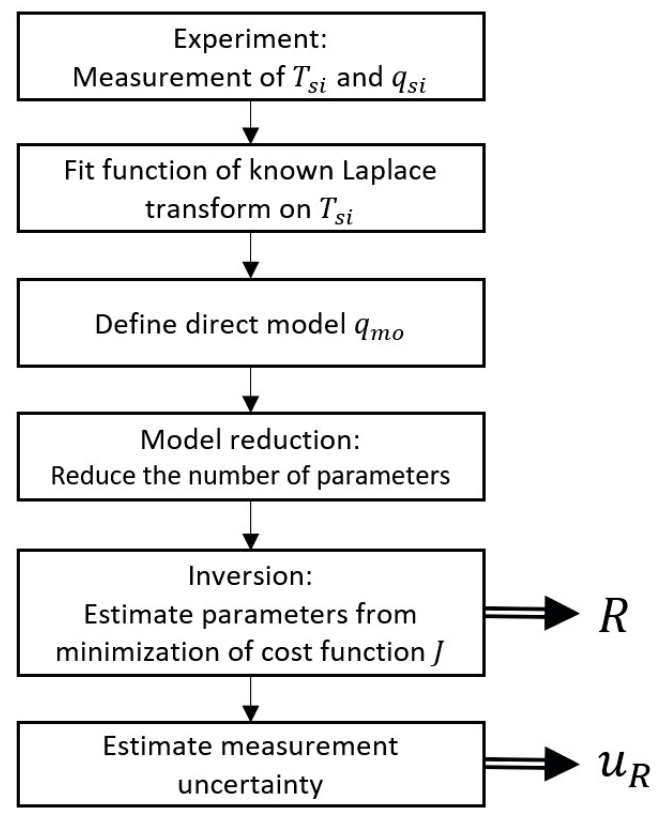

Fig. 9. Workflow of $\boldsymbol{R}$ estimation method

\section{Results and discussion}

\subsection{Result of estimation}

This section presents the results of the active method. Four consecutive experiments were carried out to assess the reproducibility of the method. One of them is presented as an example in this section. Figure 10 plots the measured surface heat flux $q_{s i}$ alongside with the model prediction $q_{m o}(\widehat{\boldsymbol{\beta}})$ after estimation of the parameters $\widehat{\boldsymbol{\beta}}$. The difference between them are the residuals. The latter are not signed (have the shape of a white noise). This is a prerequisite for the parameter estimation being correct. It proves that the model can reconstruct the measurements: the only remaining difference is the measurement noise.

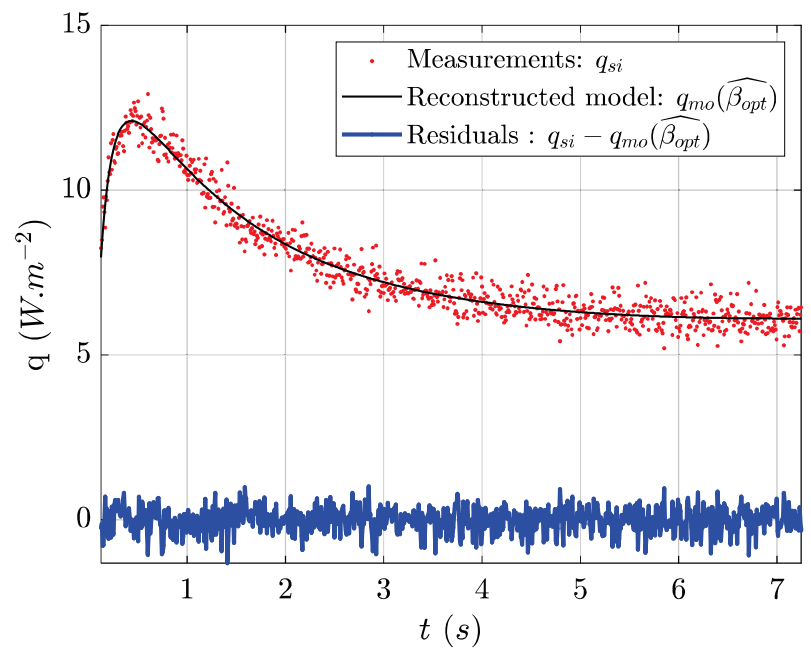

Fig. 10. Reconstruction of heat flux from the reduced model
The first few points were removed for the estimation procedure. Indeed, the changes in $q_{s i}$ are very fast at the beginning (just after the heaters are turned on). Not only does the HFM has a response time of a few minutes, but the heat flux is also very sensitive to the thermal properties of the first layer.

The estimated parameters $\widehat{\boldsymbol{\beta}}$ and their uncertainty are summarized in Table 3.

Table 3. Estimated parameters from one experiment

\begin{tabular}{|c|c|c|c|c|}
\hline $\begin{array}{c}\text { Para- } \\
\text { meter }\end{array}$ & Unit & $\begin{array}{c}\text { Reference } \\
\text { value }\end{array}$ & $\begin{array}{c}\text { Estimated } \\
\text { value }\end{array}$ & Uncertainty \\
\hline$R_{1}$ & $\mathrm{~m}^{2} \cdot \mathrm{K}^{\mathrm{W}} \mathrm{W}^{-1}$ & 0.06 & 0.05 & 0.01 \\
\hline$b_{1}$ & $\mathrm{~J} \cdot \mathrm{K}^{-1} \cdot \mathrm{m}^{-2} \cdot \mathrm{s}^{-1 / 2}$ & 420 & 421 & 24 \\
\hline $\boldsymbol{R}_{\mathbf{2}}$ & $\mathbf{m}^{2} \cdot \mathbf{K} \cdot \mathrm{W}^{-1}$ & $\mathbf{3 . 1 2}$ & $\mathbf{3 . 0 4}$ & $\mathbf{0 . 0 5}$ \\
\hline$b_{2}$ & $\mathrm{~J} \cdot \mathrm{K}^{-1} \cdot \mathrm{m}^{-2} \cdot \mathrm{s}^{-1 / 2}$ & 25 & 35.9 & 3.5 \\
\hline$b_{\text {inf }}$ & $\mathrm{J} \cdot \mathrm{K}^{-1} \cdot \mathrm{m}^{-2} \cdot \mathrm{s}^{-1 / 2}$ & 1500 & \multicolumn{2}{|c|}{ Not estimated } \\
\hline
\end{tabular}

The quantity of interest, $R_{2}$, was well estimated: a relative difference of $2 \%$ is observed when compared to the reference value given in Table 1 . The gypsum thermal resistance is estimated with a higher relative difference (10\%) but its contribution to the total thermal resistance is almost negligible so this is not a problem. The effusivity of the gypsum layer $b_{1}$ was also well estimated whereas the one of the glass wool $b_{2}$ was biased. This is due to the very low reduced sensitivity of $b_{2}$ when compared to that of $b_{1}$ (see Figure 7).

Consequently, on the presented example, the estimation of the thermal resistance of the insulation system (gypsum + glass wool) is $3.09 \mathrm{~m}^{2} . \mathrm{K}^{-\mathrm{W}^{-1}} \pm 0.06 \mathrm{~m}^{2} . \mathrm{K}^{-\mathrm{W}^{-1}}$.

\subsection{Repeatability}

The repeatability of the method was assessed by repeating the same experiment four times. Table 4 presents the estimated thermal resistance $R=R_{1}+R_{2}$ of the insulation system for each experiment.

The standard deviation between the different experiments is small: around $1 \%$. This is in good agreement with the $2 \%$ relative uncertainty predicted by the Vcor matrix (see Equation 18). The mean value is:

$R_{\text {active }}=3.10 \mathrm{~m}^{2} \cdot \mathrm{K} \cdot \mathrm{W}^{-1} \pm 0.06 \mathrm{~m}^{2} \cdot \mathrm{K} \cdot \mathrm{W}^{-1}$

which is slightly below the reference value $\mathrm{R}_{\text {passive }}=3.15 \mathrm{~m}^{2} \cdot \mathrm{K} \cdot \mathrm{W}^{-1}$ (see Section 3.1). The difference is not substantial (smaller than the uncertainties) and deemed not significant. 
Table 4. Repeatability results

\begin{tabular}{|c|c|}
\hline Experiment & $\begin{array}{c}\text { Estimated } \\
R_{1}+R_{2} \\
\left(\mathrm{~m}^{2} . \mathrm{K}^{-1}\right)\end{array}$ \\
\hline 1 & 3.10 \\
\hline 2 & 3.08 \\
\hline 3 & 3.11 \\
\hline 4 & 3.09 \\
\hline \hline $\begin{array}{l}\text { Mean } \\
\text { deviation }\end{array}$ & $\mathbf{3 . 1 0}$ \\
\hline
\end{tabular}

\section{Conclusion}

Thermal insulation of buildings has a key role to play in the reduction of greenhouse gases emissions. The socalled "performance" gap was widely documented in the literature: real thermal performances of buildings are usually very different from theoretical calculations. From this perspective, there is a need for a fast and reliable in situ measurement method of building walls thermal resistance. This paper introduces a novel active technique. Unlike passive approaches, active methods do not rely on steady-state assumptions and are less sensitive to variations of the outdoor environment. In addition, a measurement can be made within a few hours.

The proposed method was tested on a $3.2 \times 2 \mathrm{~m}^{2}$ loadbearing wall built inside a climate chamber. The wall was made of concrete building blocks and the indoor surface was covered with an internal insulation system. The thermal resistance of the insulation was first characterized in steady-state according to ISO 9869-1 [4]. The measured resistance was of $3.15 \mathrm{~m}^{2} . \mathrm{K}^{-\mathrm{W}^{-1}}$ and was used as a reference value for the validation of the active method. There was only a $1 \%$ discrepancy between this value and theoretical predictions based on materials thermal properties.

The method developed is based on simultaneous measurements of the indoor surface temperature and heat flux during an active experiment in which electric heaters powered for several hours. The direct model used in the inverse method is $1 \mathrm{D}$ and based on the thermal quadrupole formalism. Several steps of model reduction were required to limit the number of parameters for the inversion to be possible. The retained model has only three unknown parameters.

The results of the active method were good: the thermal resistance of the insulation system was estimated to $3.10 \mathrm{~m}^{2} . \mathrm{K} . \mathrm{W}^{-1}$ with a $2 \%$ uncertainty which was close to the reference value mentioned above. The method also has a good repeatability: the standard deviation between the measurements was around $1 \%$ (the same order as the measurement uncertainty predicted by the variancecovariance matrix).

The main limitation of the method is thermal bridging. The authors are working on a generalization of the presented method to non-homogeneous walls. This would enable to characterize walls with defects and/or irregularities.

We thank the CEREMA Nancy for making available their climate chamber for our experimental campaign.

\section{References}

1. D. Johnston, D. Miles-Shenton, and D. Farmer. Building Services Engineering Research and Technology, 36(5):614-27 (2015).

2. D. Bienvenido-Huertas, J. Moyano, D. Marín, and R. Fresco-Contreras. Renewable and Sustainable Energy Reviews, 102:356-371 (2019).

3. J. Meulemans, F. Alzetto, D. Farmer, C. Gorse, Buiding Information Modelling, Building Performance, Design and Smart Construction, 115127, Springer (2017).

4. ISO 9869-1:2018. Thermal insulation - building elements - in-situ measurement of thermal resistance and thermal transmittance - part 1: Heat flow meter method (2018).

5. ISO 9869-2:2018. Thermal insulation - building elements - in-situ measurement of thermal resistance and thermal transmittance - part 2: Infrared method for frame structure dwelling (2018).

6. S.E. Gustafsson. Review of Scientific Instruments, 62 (3): 797-804 (1991).

7. JCGM100:2008-GUM 1995 with minor corrections

8. J.V. Beck, B. Blackwell, C.R. St. Clair, Inverse heat conduction: Ill-posed problems. John Wiley \& Sons (1985).

9. D. Maillet, S André, J.C. Batsale, A. Degiovanni, C. Moyne, Thermal quadrupoles: solving the heat equation through integral transforms. John Wiley \& Sons Inc, (2000).

10. F.R. De Hoog, R. Frank, L.H. Knight, A.N. Stokes, SIAM Journal on scientific and Statistical Computing, 3(3), 357-366 (1982)

11. H. Orlande, O. Fudym, D. Maillet, R.M. Cotta, Thermal Measurements and Inverse Techniques, CRC Press (2011). 